\title{
Primary health care service for long-stay psychiatric in-patients
}

\author{
Nigel Fisher and Judy Roberts
}

\begin{abstract}
Aims and method A retrospective review of the activity of a general practitioner employed specifically to provide a primary health care service to the residents of an 'old long-stay' psychiatric ward was undertaken.

Results All but one patient had a chronic physical health problem requiring active management. Presenting pathology covered all organ systems. The review indicated that these health problems had not previously been satisfactorily managed by psychiatrists. This service was acceptable to and valued by patients. Clinical implications The success of the service was only achieved by dedicating specific resources to meet primary health care needs. The extent to which this model could be extended to 'new long-stay' populations located in dispersed hospital hostels is less certain.
\end{abstract}

The increased morbidity and mortality from physical health problems among those with long-term mental health problems is well documented (Honig et al, 1989). That these physical health needs may be unmet is also recognised (Brugha et al, 1989; Honig et al, 1989). As a result, approaches to meeting these needs for non-hospitalised patients are being developed, with an emphasis on facilitating access to primary health care services (Kendrick et al, 1991). Such an approach for long-stay hospital patients is problematic as after one year these patients would no longer be considered to fall under the remit of general medical services and are typically removed from their general practitioner's (GP's) list. In practice, the long-stay hospital population is unable to access ordinary primary health care services. Although dwindling, the large hospital site long-stay population remains significant at around 20000 . For these people it is assumed that the secondary mental health service is willing and able to provide a primary health care service.

This assumption is likely to be ill founded. In most services this role is left to the hospital psychiatrist, who has minimal training and no continuing education in the area. Not only are individual psychiatrists inadequately qualified but the hospital infrastructure is not geared towards primary health care: six-monthly changes of juniors mitigate against continuity of care, time spent dealing with physical health problems is often at the expense of psychiatric care. Furthermore, hospitals do not have the systems to implement screening programmes, such as cervical smears and mammography.

In an attempt to overcome these difficulties we report a service, now in operation for four years, in which a GP was specifically employed for two sessions a week to provide a primary health care service to the residents of a long-stay unit for people who have spent much of their lives in hospital.

\section{Setting}

The unit consisted of two linked wards on a large mental hospital site. The patients were drawn from a larger group of elderly long-stay patients who had been resettled as part of a previous hospital closure programme. At the start of the period there were 19 men and 25 women resident, with a mean age of 67 (range 51-85) and 78 (range 54-106) respectively. The mean length of current admission was 30 years (range 32-64). Seventy per cent of the residents had a diagnosis of schizophrenia. During the four-year period there were, on the male ward, seven new admissions and 10 deaths. On the female ward there were seven new admissions, three discharges and 11 deaths.

\section{Remit of the GP}

The GP's job covered two areas.

Assess and manage acute and chronic physical disorders

This accounted for approximately $60 \%$ of the workload. Problems reflected those of the elderly population in general. It was expected that most problems would be dealt with by the GP, with referral to the relevant specialist services as appropriate. Emergencies presenting when the GP was not present were dealt with by the ward or duty doctor. Central to the management of chronic diseases was the compilation of a 
summary of past and current physical health problems.

Provide a regular review and screen of physical health including Health of the Nation targets

A system for an annual screening medical examination and review was established. Although this would not be desirable in the general population, it was considered to be useful because of under-reporting of symptoms and high morbidity in this patient group. The medical assessment was combined with annual assessments by visiting optician. dentist and chiropodist. The screening followed that described by Kendrick (1993) and the Health of the Nation (Department of Health, 1991) targets. Included with a physical examination was a review of blood pressure, pulse, urinalysis, weight, alcohol and smoking habits. In addition, attention was paid to joint problems, skin condition, feet, oral health, vision and hearing and continence of bowels and urine.

\section{Activity}

The GP attended twice a week. In consultation with the nursing staff a schedule for routine examinations was drawn up. Any acute presentations would also be seen by a doctor for a physical health problem, including emergencies, on seven occasions per year (range 1-20). Ninety-three per cent of these contacts were by the GP.

Presenting pathology covered all organ systems (see Table 1). All patients agreed to be examined (although one took over three years of persuasion). Additionally all women had breast examinations and all but one had a cervical smear.

Table 1. Range of pathology dealt with by general practitioner

\begin{tabular}{lcc}
\hline System & $\begin{array}{c}\text { Number of } \\
\text { presentations }\end{array}$ & $\begin{array}{l}\text { Number of } \\
\text { specialist referrals }\end{array}$ \\
\hline Cardiovascular & 16 & 6 \\
Gastrointestinal & 13 & 7 \\
Endocrine & 7 & 5 \\
Skin & 11 & 0 \\
Eyes & 3 & 3 \\
Musculoskeletal & 20 & 7 \\
Gynaecological & 2 & 1 \\
Neurological & 5 & 4 \\
Respiratory & 3 & 0 \\
Ear, nose and throat & 16 & 4 \\
Renal & 5 & 2 \\
Over prescribing & 12 & 0 \\
Total & 113 & 39 \\
\hline
\end{tabular}

None of these screenings had been previously completed. All but one patient had some form of chronic physical health problem requiring active management.

One of the most common problems was over prescribing of drugs, particularly diuretics, analgesics and laxatives. Half of the patients had significant changes in management or had new problems diagnosed by the GP. During the period 21 patients died, compared to 25 in the four years prior to the GP appointment.

\section{Value of the service}

The extent and range of problems dealt with would seem to make the need for such a service self-evident. The service was certainly valued by the patients, all of whom developed a trust in the GP and allowed intimate physical examinations to take place. This was not the case when ward doctors changed every six months. Furthermore, all patients now have accessible summaries of their physical health problems and have their physical health regularly reviewed. On a 'new long-stay' unit in the same hospital, these standards were met in less than half the residents, and have deteriorated in the three years they have been monitored (Perkins. 1996).

\section{Comment}

We believe that this service has added to the quality of care of this group of long-stay patients. At a time of a primary health care-led NHS, where the Department of Health emphasises physical health as an outcome measure for people with schizophrenia (Jenkins, 1990), it is striking how the old long-stay population has become distanced from primary health care services. We believe this situation can only be remedied by specifying dedicated sessions of a specialist primary health care physician to this most needy group of patients.

This service has been economically feasible because of the relatively large number of patients with high rates of physical morbidity that are managed on a single site. How such a service may be realistically offered to the new long-stay population who are perhaps younger, at present have fewer physical health problems and dispersed in smaller, off site, 24-hour nursed care units is less obvious.

\section{References}

BRUgha. T., Wing. J. \& SMITH, B. (1989) Physical health of the long-term mentally $11 \mathrm{l}$ in the community. Is there unmet need? British Journal of Psychiatry. 155. 777-781. 
Department of HEALTH (1991) The Health of the Nation London: HMSO.

Honig. A., POP, P.. TAN, S., et al (1989) Physical illness in chronic psychiatric patients from a community psychiatric unit. The implications for daily practice. British Journal of Psychiatry. 155. 58-64.

JENKINS, R. (1990) Towards a system of outcome indicators for mental health care. British Journal of Psychiatry. 157, 500-514.

KENDRICK. T. (1993) Care of patients with schizophrenia. British Journal of General Practice, 43, 259-260.

-. SibBald. B., BuRns. T., et al (1991) Role of general practitioners in care of long-term mentally ill patients. British Medical Journal, 302. 508-510.
PERKINS. R. (1996) Rehabilitation and Continuing Care Service Clinical Audit: 1996 Annual Report. London: Pathfinder NHS Mental Health Services Trust.

*Nigel Fisher, Consultant Psychiatrist, Rehabilitation and Continuing Care Service, Springfield Hospital, 61 Glenburnie Road, London SW17 7DJ and Judy Roberts, General Practitioner. The Surgery, 219A Upper Tooting Road, London

*Correspondence

\title{
Satisfaction of attenders and non- attenders with their treatments at psychiatric out-patient clinics
}

\author{
Helen Killaspy, Julia Gledhill and Sube Banerjee
}

\begin{abstract}
Aims and method Non-attendance at psychiatric outpatient appointments has a substantial financial cost. and may also have clinical significance. To prevent non-attendance and formulate effective responses, its determinants need to be understood. Patient dissatisfaction with services has been suggested as a reason for non-attendance. we therefore investigated the role of patient satisfaction in attendance at psychiatric out-patients appointments. All patients booked for adult psychiatric out-patient follow-up appointments in a three-month period were studied using a brief, self-report questionnaire.

Results Sixty-three per cent (340/538) of attenders and $54 \%(118 / 219)$ of non-attenders responded. Responders expressed high levels of satisfaction with their treatment (92\% attenders, 91\% non-attenders) and with the service (96\% attenders, $92 \%$ non-attenders). Despite adequate statistical power, there were no statistically significant differences in satisfaction between the two groups.

Clinical implications In conclusion, patient satisfaction with psychiatric out-patient care was reported to be relatively high and did not seem to be an important determinant of non-attendance. Further work is needed to determine the impact of variables such as relapse and social disorganisation on attendance.
\end{abstract}

Developments such as the Patient's Charter have clarified the need for services to attend to user views and satisfaction. Despite innovations in service delivery and reports that home-based services can reduce non-attendance from 25 to $7 \%$ (Burns et al, 1993a), the out-patient psychiatric consultation remains the main form of contact between patients in the community and psychiatrists, perhaps due to the greater costs and time perceived to be associated with homebased services. There has, however, been little systematic study of user views of out-patient services. High non-attendance rates at psychiatric out-patient clinics of between 20 and 57\% (Baekland \& Lundwall. 1975; Carpenter et ah 1981: Morgan, 1989) have been reported, but the reasons behind this time consuming and expensive waste of scarce resources have not been investigated.

Within general medical out-patients, fixed appointment times and seeing the same doctor on successive occasions have been rated as the most important factors in consultations (Bishop et al, 1991); also patients who rate their outpatient service poorly are likely to leave their physicians (Rubin et al, 1993). Studying psychiatric in-patients. McIntyre et al (1989) reported that patients rated the ability to leave the ward when they wished and being able to talk to staff as the most important aspects of treatment. 